\title{
Supplemental Information: \\ Guidelines for publicly archiving terrestrial model data to enhance usability, intercomparison, and synthesis
}

Maegen B. Simmonds ${ }^{1,2}$, William J. Riley ${ }^{1}$, Deborah A. Agarwal ${ }^{3}$, Xingyuan Chen ${ }^{4}$, Shreyas Cholia

${ }^{3}$, Robert Crystal-Ornelas ${ }^{1}$, Ethan T. Coon ${ }^{5}$, Dipankar Dwivedi ${ }^{1}$, Valerie C. Hendrix ${ }^{3}$, Maoyi Huang ${ }^{4,8}$, Ahmad Jan ${ }^{5}$, Zarine Kakalia ${ }^{1,6}$, Jitendra Kumar ${ }^{5}$, Charles D. Koven ${ }^{1}$, Li Li ${ }^{7}$, Mario Melara ${ }^{3}$, Lavanya Ramakrishnan ${ }^{3}$, Daniel M. Ricciuto ${ }^{5}$, Anthony P. Walker ${ }^{5}$, Wei Zhi ${ }^{7}$, Qing Zhu ${ }^{1}$, Charuleka Varadharajan $^{1, *}$

${ }^{1}$ Earth and Environmental Sciences Area, Lawrence Berkeley National Laboratory, One Cyclotron Road, Berkeley, CA 94720, USA

${ }^{2}$ Now at: Pivot Bio, 2910 Seventh Street, Berkeley, CA 94710, USA

${ }^{3}$ Computing Sciences Area, Lawrence Berkeley National Laboratory, One Cyclotron Road, Berkeley, CA 94720, USA

${ }^{4}$ Pacific Northwest National Laboratory, Richland, WA, USA

${ }^{5}$ Environmental Sciences Division and Climate Change Science Institute, Oak Ridge National Laboratory, Oak Ridge, TN, 37831, USA

${ }^{6}$ College of Natural Resources, University of California Berkeley, Berkeley, CA 94720, USA

${ }^{7}$ Department of Civil and Environmental Engineering, The Pennsylvania State University, State College, PA 16802, USA

${ }^{8}$ Now at: Modeling Program Division, Office of Science and Technology Integration, National Weather Service, National Oceanic and Atmospheric Administration

*Corresponding author: cvaradharajan@lbl.gov 


\section{Supplemental Information 1: Questions for assessing model data archiving needs for terrestrial modeling community}

1.1 Model data background and storage needs:

1. Name the model(s) you would like to archive data from:

2. How are your data represented spatially? (e.g., $1 \mathrm{~km}^{2}$ resolution):

3. Temporal discretization and range of dataset (e.g., hourly time-step for 100 years):

4. Number of files for a typical simulation to be archived:

5. Average file size for a typical simulation to be archived:

6. Types of file formats for a typical simulation to be archived (e.g., netCDF):

1.2 What's worth archiving, why, and for how long?

1. Which model data are worth archiving? (Rate each of the following on a scale from 1-5 as Not important at all, Not so important, Somewhat important, Very important, or Extremely important)
a. Model inputs
b. Model outputs
c. Metadata
d. Model code
e. Other scripts
f. Model testing data
g. Other: Describe any other type of model data worth archiving

2. In general how long do you think model data remains useful? (multiple choice)
a. $<1$ year
b. 1-2 years
c. 5-10 years
d. $<10$ years

3. Rate the importance of having these features in a model data repository on a scale of 1-5 (Not important at all, Not so important, Somewhat important, Very important, or Extremely important)
a. Sharing of data
b. Data preservation (for time period indicated above)
c. Complete model data packages that can reproduce the model outputs
d. Clear documentation
e. Usability of archived model data

4. Describe any additional considerations that are important to informing the development of a successful model data archive.

\subsection{Approaches to archiving model data}

1. Does your group currently archive model data? (yes or no)

2. Describe your group's approach to archiving model data.

3. Would you be willing to share documentation of your model data archiving protocol?

4. How satisfied are you with your model data archive? (0: Very dissatisfied to 5: Very satisfied)

\subsection{Last thoughts}

1. Note any recommendations you have for model data storage options.

2. Would your group be willing to learn a new method for data archiving? (yes, no, absolutely not, maybe)

3. Note any other thoughts or comments here. 
Supplemental Table 1. Summary of the journal articles and corresponding public model data archives curated by researchers funded by the U.S. Department of Energy. Examples of code and data availability statements are provided to assist researchers seeking to use our recommended guidelines. The references in these statements are included in the main text bibliography.

\begin{tabular}{|c|c|c|c|}
\hline $\begin{array}{c}\text { Journal } \\
\text { Article }\end{array}$ & Model & $\begin{array}{c}\text { Storage } \\
\text { location(s) } \\
\text { of public } \\
\text { data archive }\end{array}$ & Code and Data Availability statements from the journal articles \\
\hline $\begin{array}{l}\text { Walker et al. } \\
\quad(2019)\end{array}$ & $\begin{array}{l}12 \text { models used } \\
\text { in the FACE- } \\
\text { MDS (Free Air } \\
\mathrm{CO}_{2} \\
\text { Enrichment } \\
\text { Model Data } \\
\text { Synthesis) }\end{array}$ & $\begin{array}{l}\text { ESS-DIVE } \\
\text { (Data } \\
\text { repository) }\end{array}$ & $\begin{array}{l}\text { The site-based meteorological dataset (https://data.ess- } \\
\text { dive.lbl.gov/view/ess-dive-7807cf86fl } 1 \mathrm{dd42a}- \\
\text { 20181127T173047368940), the model output dataset (https://data.ess- } \\
\text { dive.lbl.gov/view/ess-dive-8260043c35fc925-20181130T171955541030) } \\
\text { and the experiment dataset (https://data.ess-dive.lbl.gov/view/ess-dive- } \\
\text { f525c71da7d2681-20181128T160851574946) generated and analyzed } \\
\text { during the current study are available at the US Department of Energy's } \\
\text { (DOE) ESS-DIVE repository. }\end{array}$ \\
\hline $\begin{array}{l}\text { Koven et al. } \\
\quad(2020)\end{array}$ & FATES & $\begin{array}{l}\text { Zenodo } \\
\text { (Data } \\
\text { repository), } \\
\text { GitHub, } \\
\text { NGEE- } \\
\text { Tropics } \\
\text { (Project } \\
\text { website) }\end{array}$ & 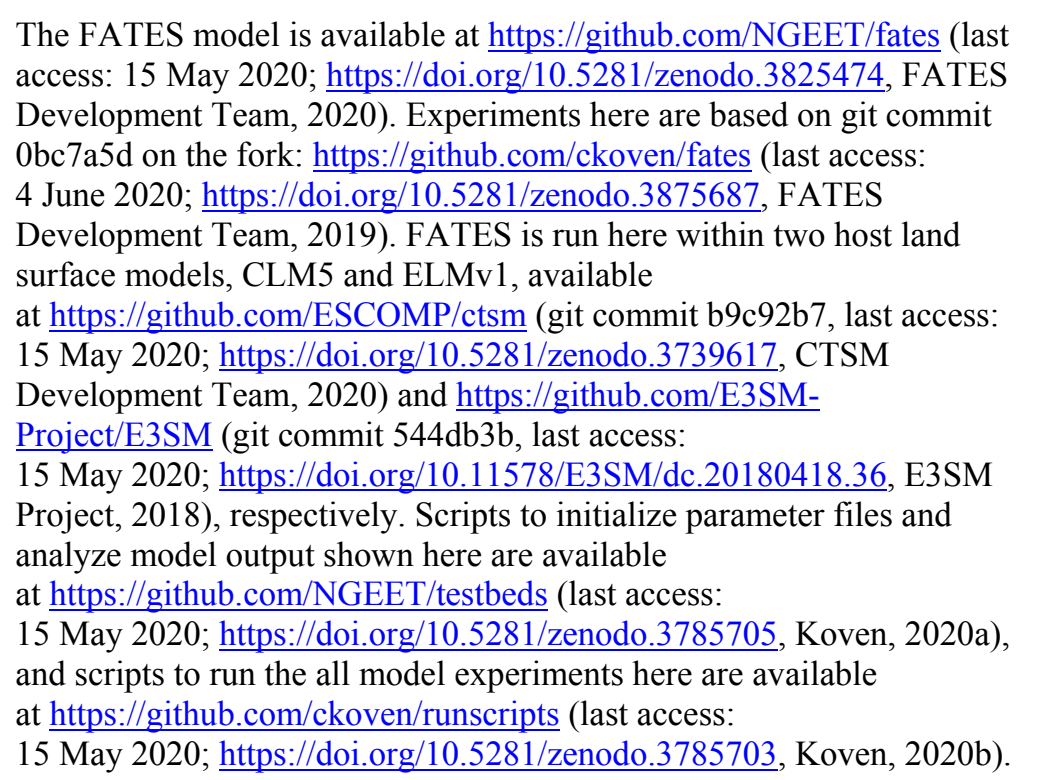 \\
\hline $\begin{array}{c}\text { Jan et al. } \\
(2020)\end{array}$ & ATS & $\begin{array}{c}\text { NGEE } \\
\text { Arctic } \\
\text { (Project } \\
\text { website) }\end{array}$ & $\begin{array}{l}\text { The Advanced Terrestrial Simulator (ATS) (Coon et al., 2019) is open } \\
\text { source under the BSD 3-clause license and is publicly available } \\
\text { at https://github.com/amanzi/ats (last access: October 2019; Coon, 2016). } \\
\text { Simulations were conducted using version 0.88. The ATS version } 0.88 \text { is } \\
\text { permanently stored at https://doi.org/10.5281/zenodo.3727209 (Coon } \\
\text { et al., } 2020 \text { ). Forcing data, model input files, Jupyter notebooks used to } \\
\text { generate figures, and meshes along with Jupyter notebooks used to } \\
\text { generate the meshes are publicly available } \\
\text { at https://doi.org/10.5440/1545603 (Jan et al., 2019). Data products used } \\
\text { in the model comparisons are publicly available through the NGEE } \\
\text { Arctic long-term data archive https://doi.org/10.5440/1416559. The } \\
\text { observed water level can be accessed } \\
\text { at https://doi.org/10.5440/1183767 (Liljedahl and Wilson., 2016), the } \\
\text { soil temperature data at https://doi.org/10.5440/1126515 (Romanovsky } \\
\text { et al., 2017), and the evapotranspiration data } \\
\text { at https://doi.org/10.5440/1362279 (Dengel et al., 2019, respectively.) }\end{array}$ \\
\hline
\end{tabular}



Zhu et al. Nutrient Dryad (Data Data associated with this paper have been deposited in a Dryad digital (2017) competition repository) repository https://doi.org/10.5061/dryad.7449j theories used in land components of Earth System Models: CT2,
CT4, and CT5 (Equilibrium Chemistry Approximation)
Zhi et al. BioRT- ESS-DIVE All data are available at data portal for East River watershed in Colorado, (2019)

Flux- (Data managed by Lawrence Berkeley National Laboratory. The original code PIHM Repository), (https://github.com/PSUmodeling/BioRT-Flux-PIHM). This website is developing, with detailed instructions and example files added over time. A model development manuscript is in preparation, to be published with information on code structure and application examples.*

\footnotetext{
* The data and code availability statement was in the Acknowledgements section
} 